\title{
Training Future Family Physicians to Become Master Adaptive Learners
}

Louito Edje, MD, MHPE; David W. Price, MD

\begin{abstract}
This article examines the use of a concept that teaches learners how to learn in the context of family medicine residency training. We describe the four phases of this master adaptive learning framework and its place in educational theory and adaptive expertise, its implications for graduate medical education training and Accreditation Council for Graduate Medical Education competencies, as well as its role in imprinting family medicine residents for career-long learning. We lay out pragmatic strategies supporting this concept with a proposed curricular format for training in family medicine, including small group teaching methods, didactics, the clinic visit, faculty development and an optimal learning environment.
\end{abstract}

(Fam Med. 2021;53(7):559-66.) doi: 10.22454/FamMed.2021.192268

Published Online First June 8, 2021

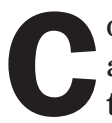
onsider you are traveling on a preset course to a destination only to encounter a problem that obstructs the road ahead. This obstruction has occurred recently enough that there is no official detour or paved exit from your location. You need to find an off-road route to continue your journey. You prepare, mapping an alternate route, and decide you need resources such as fourth gear for the unknown terrain. You learn which resources are useful in the process, and adapt to arrive at your final destination.

The ability to adapt to novel clinical problems and practice challenges is important for family physicians because more than $50 \%$ of late-career, practicing family physicians have worked in more than three different clinical practices and their patient populations have changed significantly in the process. ${ }^{1}$ Additionally, with the acceleration of new knowledge facing physicians, the ability to adapt practice to maintain expertise is a skill that ideally could be taught in residency. Like the traveler, a family physician may face clinical problems for which there are no known solutions. A clinical example of a detour is the alternate approach a learner might take for treatment of thrombosis in a patient with a concomitant, unexpected finding of thrombocytopenia. The learner may ordinarily consider as first-line therapy a certain class of thrombolytics (that has thrombocytopenia as a potential side effect), but now the learner must make a different choice to mitigate patient harm. The learner may also decide to add this alternative treatment as a new choice in his or her routine treatment compendium for all subsequent patients seen with a diagnosis of thrombosis.
The master adaptive learner (MAL) model provides an approach for learning and also aids adaptation of expertise over time. ${ }^{2}$ There is no reference to MAL and graduate medical education (GME), or MAL and family medicine (FM), in the medical education literature. Thus, incorporation of MAL into residency training is yet unexplored and unevaluated.

In this article, we differentiate between routine and adaptive expertise; discuss the phases of the MAL concept and where it fits in education theory; consider its implication for GME, particularly for Accreditation Council for Graduate Medical Education (ACGME) competencies, and overall principles; and its implications on FM GME: curriculum, didactics, clinic visits, faculty development and clinical learning environments.

\section{The MAL Concept and Theoretical Framework} Routine and Adaptive Expertise Dreyfus and Dreyfus introduced a theory on expertise involving five stages of development as a learner moves from novice toward expert. ${ }^{3}$ Ericsson believed time spent in deliberate practice in domains

From the Departments of Medical Education and Family and Community Medicine, University of Cincinnati, Cincinnati $\mathrm{OH}$ (Dr Edje); Department of Family Medicine, University of Colorado Anschutz School of Medicine, Aurora, CO (Dr Price); and American Board of Family Medicine, Lexington, KY (Dr Price). 
such as music, chess, and sports, was central to the development of expertise. ${ }^{4}$ Gobert and Chassy subsequently described a theory of intuition as a perceptual process essential to the development of expertise. ${ }^{5}$ Ultimately, routine expertise has become defined as the mastering of performance to the level of efficiency and accuracy based on skills and knowledge learned over time. ${ }^{2}$ Routine experts efficiently address problems that have established solutions. In comparison, adaptive experts balance routine expertise in straightforward situations with the use of innovative problem solving in response to novel practice challenges. ${ }^{6}$ For example, clinical flexibility of the family physician was celebrated in response to the first surge of the COVID-19 pandemic, as clinics transitioned to either all virtual or hybrid models of delivering care within weeks, while maintaining high levels of patient satisfaction. ${ }^{7}$ Adaptive experts explore new concepts, ask questions which often lack known answers, invent new solutions, and are prepared to remain curious and effectively self-regulate their learning to proactively meet the changing needs of their future practice. ${ }^{8}$

\section{The MAL Concept}

The MAL model is grounded in selfregulated learning theory, providing a foundation for adaptive expertise.
MALs go through four distinct phases: planning, learning, assessing, and adjusting. The model aligns with concept of practice-based learning and improvement and the plan-dostudy-act (PDSA) cycle (Table 1). ${ }^{9}$

In the planning phase, learners identify knowledge gaps, heralded by what Schon described as a surprise, or Mezirow as a disorienting dilemma-an uncomfortable feeling of not knowing. ${ }^{10-12}$ This cognitive dissonance is a key motivator for individuals to seek opportunities to address those gaps. Goal-setting and prioritization is critical as they search for learning resources. This is the time learners are most receptive to learning. ${ }^{13}$

Once resources have been selected, the learning phase occurs as new knowledge is used to decrease discomfort created by the previously identified gaps. Careful selection of evidence-based material is critical to this phase. During the third phase, learners determine if what was learned is useful. It is well established that physicians' self-assessment abilities are weak. ${ }^{14,15}$ But, informed self-assessment, which incorporates objective data and external assessment, is more reflective of the learner's performance. Sargeant notes that a complex interplay of external and internal factors as well as tension within learners and the learning environment determine whether individuals choose to ignore, reject, seek, or accept input from an assessor. Thus, feedback must be "clear, timely, specific, constructive, and preferably offered by trusted, credible supervisors in a safe environment, to inform a clinician's selfassessment." 16

During the fourth phase (adjusting), learners incorporate what has been learned into practice, deciding if this newly learned information should be applied to a single instance or if it is widely applicable to more than one patient or process.

Price articulated an adaptive cycle of continuing professional development for physicians that aligns with the PDSA model of quality improvement. ${ }^{17}$ This model can also connect learning and doing in graduate medical settings. It can help residents function as part of their health system during their training and enable them to develop lifelong learning and improvement skills adaptable to a number of different practice systems or settings across their careers. It can thus be considered an adaptive cycle of continuous lifelong learning and improvement. At the point of care, a family physician (or resident) may experience a surprise of disorienting dilemma that identifies a knowledge or skill gap that could include requirements for improvement of knowledge ("knows what to do"), competence

Table 1: MAL Phase and Strategy Aligned With Plan-Do-Study-Act

\begin{tabular}{|l|l|l|}
\hline \multicolumn{1}{|c|}{ MAL Phase } & \multicolumn{1}{|c|}{ Plan-Do-Study-Act } & \multicolumn{1}{c|}{ MAL Strategy } \\
\hline Planning & Plan & $\begin{array}{l}\text { Questioning } \\
\text { Prioritizing } \\
\text { Goal-setting }\end{array}$ \\
\hline Learning & & $\begin{array}{l}\text { Critical appraisal } \\
\text { Knowledge retrieval } \\
\text { Spaced repetitious learning } \\
\text { Collaboration } \\
\text { Elaboration } \\
\text { Concept interleaving }\end{array}$ \\
\hline Assessing & Do & $\begin{array}{l}\text { Self-assessment } \\
\text { External feedback }\end{array}$ \\
\hline Adjusting & & $\begin{array}{l}\text { Individual } \\
\text { System }\end{array}$ \\
\hline
\end{tabular}

Abbreviation: MAL, master adaptive learner. 
("knows how to do"), or conditional knowledge ("knows when to do"). In the example of the traveler, the disorienting dilemma occurred as the traveler arrived at the obstruction on the highway. The traveler is in an unexpected situation and must reorient and adapt to move forward. This could be done through reading, consulting peers, or other more formalized educational activities. After deciding on a course of action and applying it to the care of the patient, patient data and subsequent progress is used as an indicator of progress. This cycle could continue until the patient reaches the desired outcome; the physician can then decide if the learnings should be more broadly applied to other patients. At the practice level, need for improvement in one's professional practice can be identified from one or more sources, including quality process or outcome metrics, population health data, peer or patient surveys, patient safety events, other sources of big data, or recent tests of cognitive expertise. ${ }^{18}$ If improvement opportunities are identified, causes of the gap between current and desired performance could be explored, and opportunities for addressing the gap can be developed and addressed through mechanisms similar to those mentioned above. Learnings could then be implemented in practice, by systematically using structured practice improvement activities. Data would then be used to assess the outcomes of learning, and the cycle could be repeated until improvement goals have been achieved. This type of feedback can inform health systems improvement as well as individual improvement. Figure 1 shows how this adaptive cycle of lifelong learning and improvement aligns with concepts of MAL.

Though two different processes, this shows continuing professional development's close alignment with MAL. They provide models for learning in residency that can be used beyond formal training.

\section{Learning After Formal Training}

The formal training segment of a family physician's carreer, through completion of GME, is highly structured and carefully monitored (Figure 1). ${ }^{17,19}$ Learning is context-dependent and less standardized after formal medical training.

Evidence suggests many clinicians may not be learning effectively in practice. ${ }^{8,20}$ Ross et al have recently demonstrated that critical thinking skills in practicing family physicians are not as strong as those of residents, as evidence by lower scores on the California Critical Thinking Skills Test (CCTST). ${ }^{21}$ Individual learning paths are not linear and may include regression. Experienced practicing physicians may return to the novice level as new knowledge is introduced or new conditions such as the COVID-19 pandemic arise.

\section{How MAL Applies to GME}

GME is an ideal stage of medical training in which to use the concept of MAL because educational imprinting occurs in formative clinical experiences. ${ }^{22}$ The ACGME/American Board of Medical Specialties (ABMS) competencies of medical knowledge, practice-based learning and improvement, system-based practice,

Figure 1: Continuous Lifelong Learning and Improvement and the Master Adaptive Learner

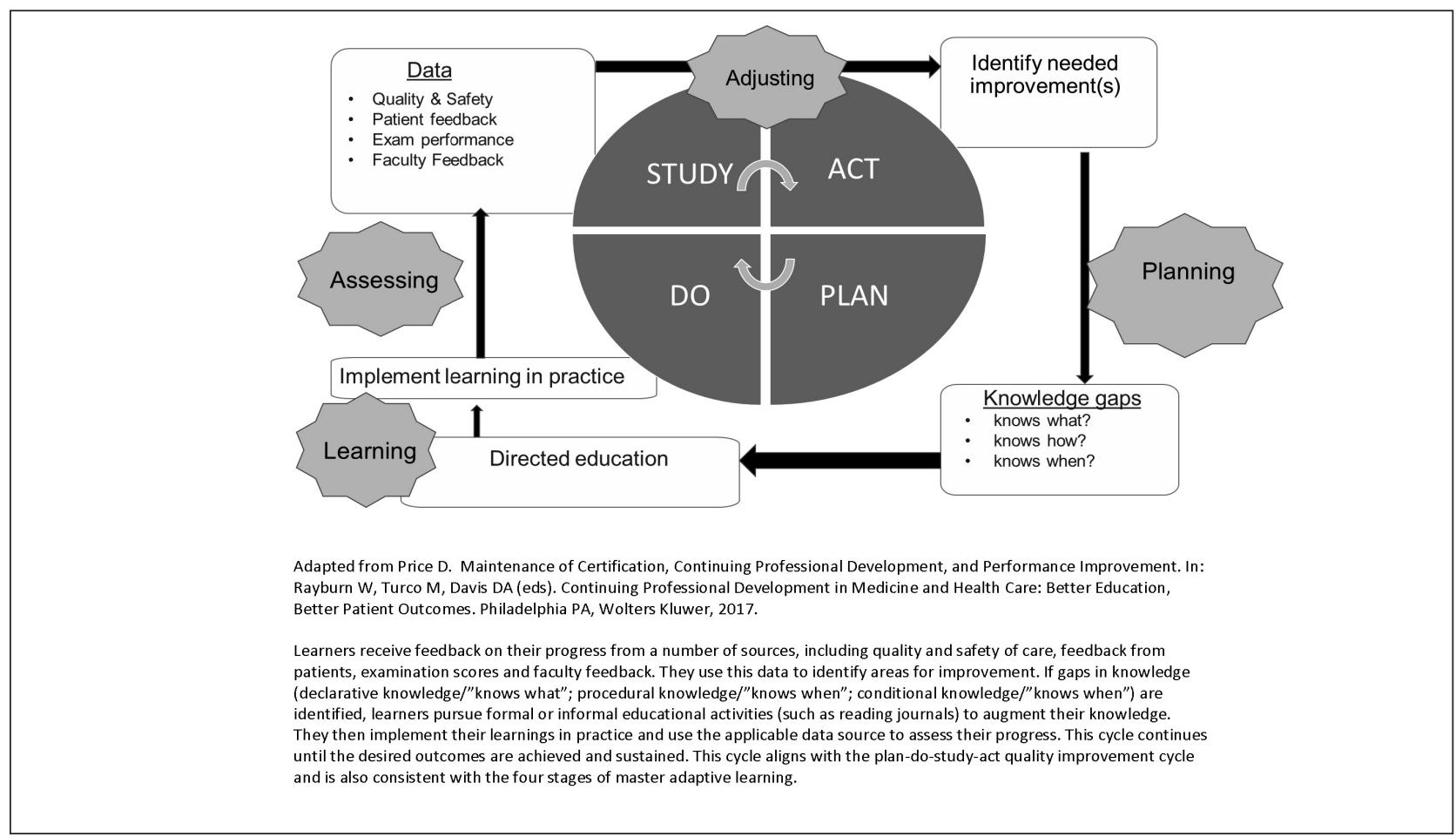


interpersonal and communication skills, and professionalism remain relevant for the family physician of the future, however specific overlaps between current competencies may be needed to support MALs. With the pace at which new knowledge is generated, consideration should also be given to a new competency of master adaptive learning. This competency could be assessed and included in the overall demonstration of readiness to practice independently. Table 2 illustrates how the MAL concepts align with these competencies.

\section{The Implications of MAL for Family Medicine GME} The MAL Curriculum

Teaching strategies that emphasize metacognition (an awareness of one's own thought processes), critical thinking, and self-reflection promote the MAL conceptual framework. MAL should not be taught just as a block didactic. Rather, the MAL concept could be introduced early in residency, and continually developed through individual and group learning. Learners then revisit, apply, and refine these skills to situations with increasing levels of complexity as they progress through training. Faculty can help signpost the use of the model by reminding residents of opportunities to apply it in different situations and settings (Figure 2). Curiosity can be maintained by connecting previous experiences to new problems and making them personally relevant to the learner. ${ }^{30,31}$ Additionally, curiosity is enhanced with introduction of new opinions. ${ }^{32}$

Group learning can utilize either problem-based learning (PBL) or team-based learning (TBL). PBL most closely aligns with the MAL concept, ${ }^{27,33}$ with planning, learning, and assessing as key components. In this format, team-identified learning objectives are used as individuals

Table 2: ACGME/ABMS Competencies in the Context of MAL

\begin{tabular}{|c|c|}
\hline ACGME/ABMS Core Competencies & MAL \\
\hline Patient care & $\begin{array}{l}\text { There will be an ongoing need for patient care competency } \\
\text { across a spectrum of care settings and condition acuities. } \\
\text { Family physician MALs will need to efficiently traverse } \\
\text { from one area of expertise to another, and adjust their care } \\
\text { of patients as their care settings and population(s) change } \\
\text { over time. }{ }^{23} \text { They would be trained to effectively self-regulate } \\
\text { their learning, aggressively seek and fill their patient care } \\
\text { gaps as a matter of routine, and engage in the critical } \\
\text { reflection required to continuously prioritize and update } \\
\text { understanding of new practice challenges. }\end{array}$ \\
\hline Medical knowledge & $\begin{array}{l}\text { Medical knowledge would include mining, analyzing, } \\
\text { interpreting and ethically utilizing big data, artificial } \\
\text { intelligence, and data gathered by tools such as wearable } \\
\text { technologies. Family physicians would effectively and } \\
\text { efficiently self-regulate learning as a multitude of new } \\
\text { diseases and treatments are continually discovered. Because } \\
\text { critical thinking skills do not currently seem to improve } \\
\text { during residency, }{ }^{20} \text { we could teach, and explicitly assess, } \\
\text { critical thinking skills. }\end{array}$ \\
\hline Systems-based practice & $\begin{array}{l}\text { Systems-based practice would include health system } \\
\text { science. We would prepare family physicians to be involved } \\
\text { in medical staff structure and function to provide a } \\
\text { primary care, patient-centered perspectives to gap-analyses } \\
\text { performed by health systems for quality improvement, } \\
\text { patient safety and enhanced patient experience. Formal } \\
\text { training in personal and organizational leadership would } \\
\text { be taught to facilitate MALs' interactions with inter- } \\
\text { professional teams. It has been long established that access } \\
\text { to primary care is associated with improved health outcomes } \\
\text { and decreased cost, yet } 30 \% \text { of the US population has } \\
\text { difficulty accessing primary care physicians. The percentage } \\
\text { of family physicians providing care across all health care } \\
\text { settings declined } 26 \% \text { between } 2013 \text { and } 2019 \text {. There is a } \\
\text { declining presence of family physicians in hospital-based } \\
\text { care. }{ }^{24-26}\end{array}$ \\
\hline
\end{tabular}


Table 2: Continued

\begin{tabular}{|c|c|}
\hline ACGME/ABMS Core Competencies & MAL \\
\hline Practice-based learning and improvement & $\begin{array}{l}\text { Practice-based learning and improvement is consistent } \\
\text { with lifelong learning }{ }^{27} \text { and perhaps the most intuitively- } \\
\text { aligned competency with the MAL framework. Formal } \\
\text { training during undergraduate and graduate medical } \\
\text { education should prepare MALs to efficiently and effectively } \\
\text { learn throughout their careers. Practice-based learning } \\
\text { and improvement should enable critical reflection, clinical } \\
\text { reasoning and informed self-assessment }{ }^{27} \text {-all critical to the } \\
\text { development of MALs. } \\
\text { Learners need just-in-time access to personal practice } \\
\text { data enabling indicators of gaps and opportunities for } \\
\text { improvement. Learners need to be taught how to access, } \\
\text { analyze, and interpret this data. }\end{array}$ \\
\hline Professionalism & $\begin{array}{l}\text { Professionalism would emphasize timely and meaningful } \\
\text { responsiveness to needs of patients, teams, systems and } \\
\text { populations. This includes advocacy of individual patients } \\
\text { in their own care as well as advocacy of populations facing } \\
\text { crises, such as the Flint Water crisis. }{ }^{28} \text { The MAL would } \\
\text { be trained in to function in interdisciplinary teams which } \\
\text { interact with patients in all settings in which they receive } \\
\text { care regardless of the physician's ability to be physically } \\
\text { present. }\end{array}$ \\
\hline Interpersonal skill and communication & $\begin{array}{l}\text { Interpersonal communication skills would be developed } \\
\text { and assessed by real time interactions, use of high- } \\
\text { fidelity simulation, and feedback from patients of various } \\
\text { racial, ethnic, socioeconomic, gender identity and religious } \\
\text { differences. This would enable the development of cultural } \\
\text { humility and prepare future family physicians to recognize } \\
\text { and address health care disparities and the social } \\
\text { determinants of health in the communities in which they } \\
\text { will work. }{ }^{29} \text { The MAL will need to understand, for example, } \\
\text { what it is like to try to exercise in an unsafe community } \\
\text { and understand challenges of maintaining a healthy diet } \\
\text { while living in a food desert. The curriculum would include } \\
\text { social justice and provide interfaces for learning within the } \\
\text { patient's community to cultivate trust which may facilitate } \\
\text { participation of marginalized populations in their care and } \\
\text { in clinical research. }\end{array}$ \\
\hline
\end{tabular}

Abbreviations: ACGME, Accreditation Council for Graduate Medical Education; ABMS, American Board of Medical Specialties; MAL, master adaptive learner.

engage in self-study on a particular topic or case, then convene in small groups to apply their learning. An example of PBL could include managing blood pressure in an elderly patient with renal disease. Objectives may include choice of safest medication and dose. Team members would individually decide how they would manage, then reconvene and discuss with the small group. Curiosity and growth mindset are also cultivated in PBL. ${ }^{34}$ In TBL, learners work on the same problem together from the outset. In either format, smaller group size enables individualized faculty-learner interaction as well as peer feedback. The MAL concept can be applied in response to individual learning needs that are identified while caring for patients or populations of patients. Both teaching methods are didactic in nature and can be incorporated into specific curricula. For example, if a residency had "Cardiology Tuesdays" during which the focus of didactic learning would be cardiology, both PBL and TBL could be used to engage learners to approach unexpected cardiac problems with novel solutions. These methods are conducive to multiple phases of MAL.

\section{MAL Concept in the Clinic Visit}

The family practice clinic visit is the optimal learning environment for MALs. Well-trained faculty would be able to signpost and identify teachable moments while creating a safe space in which learners are challenged to activate the MAL cycle. Observation of learner skills, with timely feedback, may contribute to the external component in the informed self-assessment phase 
Figure 2: MAL Curriculum of Family Medicine Residency Education

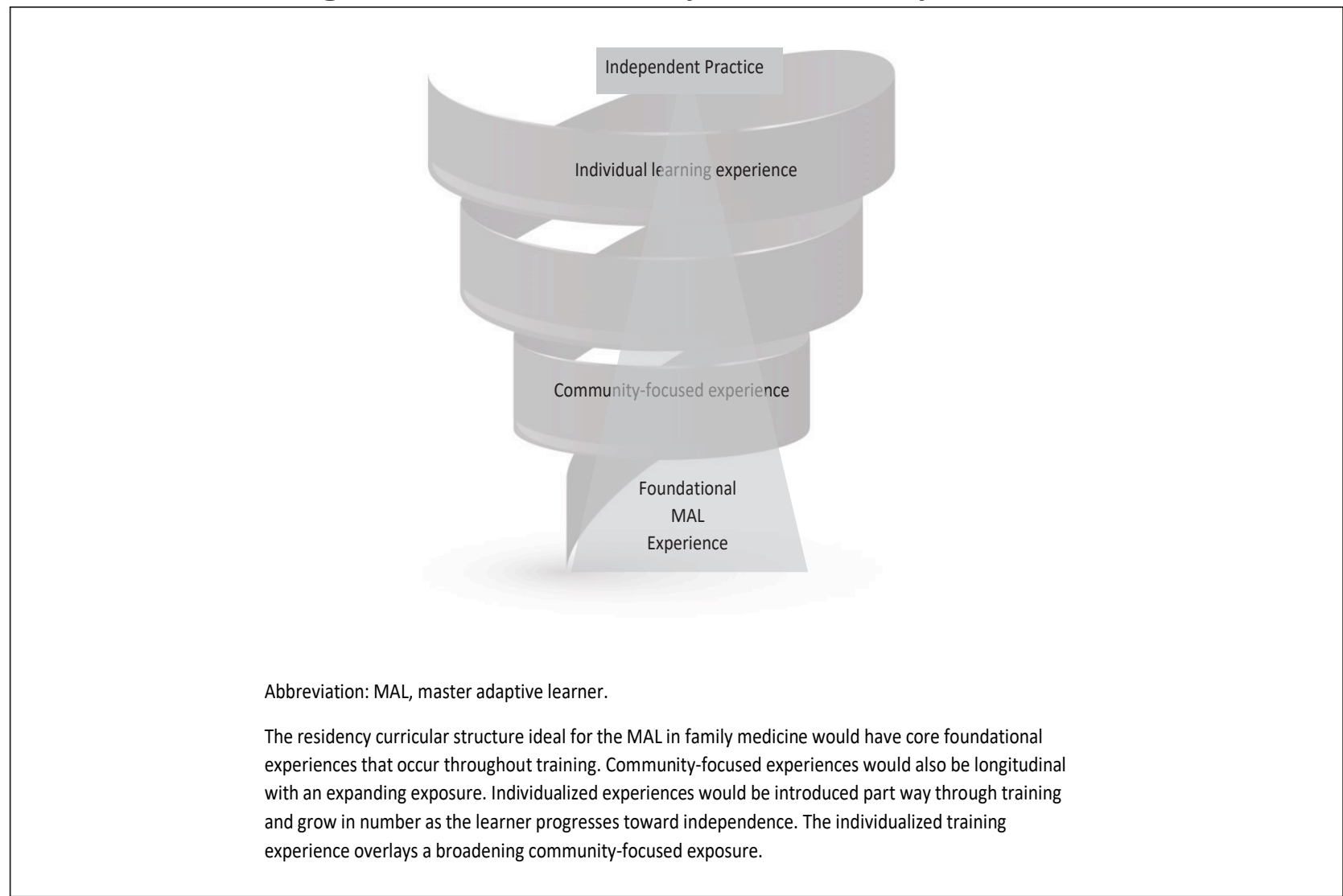

of MAL. Learners can be prompted to think aloud as they tackle both routine and unexpected clinical problems. Beyond direct clinical problem-solving, this setting also provides opportunities for faculty and upper-level residents to role-model skill acquisition. Clinical reasoning can be communicated to the learners while faculty are demonstrating specific skills. Learners can be encouraged to question the faculty during the role-modeling. For example, while demonstrating how to do a punch biopsy, if a faculty wanted to emphasize the importance of depth perception, he or she may signal the appropriate depth has been reached by stating one should feel a change in resistance of the instrument as it is entering subcutaneous tissue. The faculty may then ask what the learner thinks causes the change in resistance. The faculty may also ask the learner what he or she would do next if the change in resistance was not felt or identify an area of the body in which punch biopsies would not be recommended, such as an eyelid, where depth is critically important. Or, the faculty may ask in what other situations this procedure would be ideal aside from removing concerning skin lesions. In another example, learners can also be empowered to learn from each other. In this case, two residents may be seeing their own patients in a clinic session. Resident one is an osteopathic resident who knows how to do suboccipital release for a patient with a headache. Resident two, who has been allopathically trained, has a patient with a headache unresponsive to standard pharmacotherapy. Resident two is feeling a gap (Schoen surprise) in his ability to help the patient. It is then the resident is most ready to learn new information and techniques. ${ }^{13}$ This resident knows Resident one is in clinic and asks him/her to teach suboccipital release on his patient. Resident two now has a new skill which he/she may try on subsequent patients, as appropriate.

\section{The MAL Learning Environment}

Teachers and teaching institutions can contribute to an environment that enables MALs to thrive. Most faculty have not yet been trained in MAL; adaptive educators must therefore be selected and developed. Adaptive educators demonstrate a growth mindset, authenticity, resilience, trustworthiness, tolerance for ambiguity, expertise, humility and honesty. ${ }^{35}$ They may role-model their own acquisition of new knowledge; express their thought processes explicitly (think out loud); describe how they critically appraise literature to arrive at evidence-based decisions; signpost the use of the MAL model; set clear expectations; prompt learners to articulate their problem-solving processes; provide feedback on 
observations and allow the learner to articulate how to improve; and reward curiosity. They may support learning by helping learners thrive in interprofessional, team-based, case-based, and problem-based learning, as well as individual coaching with an emphasis on deliberate practice. Faculty development should focus on coaching more than teaching and emphasize that each learner needs to learn how he or she thinks.

Institutional leadership may integrate partners committed to the development of MALs. Institutional culture that supports MALs may value discussion, vulnerability, patient safety, accountability, teaching, ambiguity tolerance, flexible processes, closed-loop communication, and quality improvement. Resources supporting MALs should be both human and material, including considerations of time, space, data, adaptive educator development, and medical informaticists. Institutional and residency leadership could facilitate MAL by acknowledging the need to explore workflow-based strategies to allow time for critical reflection as part of a normal work day. An organization's transparency in responses to medical errors can set a tone for MALs regarding the receptiveness to learn from mistakes. This, in turn, can create an environment in which learners feel like valueadded members of care teams who are comfortable identifying personal and institutional gaps and learning to fill those gaps with appropriate guidance.

GME communities within health systems should welcome MALs and provide adaptive learning environments commensurate with their level of progression through training. ${ }^{36}$ MALs who have been well supported in the undergraduate medical education environment should be received into the next level of formal training by adaptive educators who are familiar with a culture of curiosity in which vulnerability is embraced; where there is receptiveness to feedback and nurturing of new ideas that challenge existing knowledge.

\section{Conclusion}

Testing and evaluation is needed on incorporation of MAL concepts into FM residency education. Piloting and assessing ways to allow time for critical reflection as part of workflow could be promising areas for GME scholarship.

The MAL model is ideal for the core values that ground family physicians in a rapidly changing environment. For the MAL model to be effective, it must reside in a health care ecosystem of engaged stakeholders beyond core FM graduate medical educators. They should understand and appreciate the MAL model and its implications for developing a highly skilled workforce. MALs are well suited to improve processes within learning organizations. Real-time or just-in-time access to practice data is needed so learners can receive external indicators of their gaps and fill those appropriately. This environment would also need to foster curiosity, teaching on how data could be mined, analyzed, interpreted, and utilized by learners. A MAL-centric institutional culture should reflect a fearless, nurtured curiosity. The tenets of such organizational culture should be made standards of institutional accreditation.

By the time they leave training, MALs should have gained deep conceptual and practical knowledge and skills so they are well prepared for future learning. These skills should be durable across practice models and health care ecosystems. This does not imply family physicians should leave training "knowing everything." Rather, MAL skills should enable family physicians to effectively and efficiently learn as they encounter new patient and community needs and as their practices change and mature over time. As MALs, they will enhance their contributions to the interprofessional, team-based care they deliver to all their patients. It is important that our trainees are able to demonstrate FM core values, remain pluripotent, and adapt, so they are able to consistently provide high-quality care in any number of teams, systems, and communities over the course of their career.

CORRESPONDING AUTHOR: Address correspondence to Dr Louito Edje, University of Cincinnati Medical Center, 234 Goodman Street, Suite 1320, Cincinnati, OH 45219. 513584-4118. edjelc@ucmail.uc.edu.

\section{References}

1. Community Dialog: Survey Results (Late Career Diplomates). ABFM Starfield Summit IV. https://residencysummit.squarespace.com/ community-dialogue. Accessed March 21, 2021.

2. Cutrer WB, Miller B, Pusic MV, Mejicano G, Mangrulkar RS, Gruppen LD, Hawkins RE, Skochelak SE, Moore DE Jr. Fostering the development of master adaptive learners: a conceptual model to guide skill acquisition in medical education. Acad Med. 2017 Jan;92(1):70-75. doi: 10.1097/ACM.0000000000001323.

3. Dreyfus S. The five-stage model of adult skill acquisition. Bull Sci Technol Soc. 2004;24(3):177181. doi:10.1177/0270467604264992

4. Ericsson A, Krampe RT, Tesch-Römer C. The role of deliberate practice in the acquisition of expert performance. Psychol Rev. 1993;100(3):363-406. doi:10.1037/0033295X.100.3.363

5. Gobet F, Chassy P. Expertise and intuition: a tale of three theories. Minds \& Machines. 2009. 19:151-180. doi: 10.1007/s11023-008-9131-5

6. Mylopoulos M, Regehr G. Cognitive metaphors of expertise and knowledge: prospects and limitations for medical education. Med Educ. 2007;41(12):1159-1165. doi:10.1111/j.13652923.2007.02912.x

7. Olayiwola JN, Magaña C, Harmon A, et al. Telehealth as a bright spot of the COVID-19 pandemic. JMIR Public Health Surveill. 2020;6(2):e19045. doi:10.2196/19045

8. Regehr G, Mylopoulos M. Maintaining competence in the field: Learning about practice, through practice, in practice. J Contin Educ Health Prof 2008; 281 suppl1):S19-S23.

9. Langley G, Moen R, Nolan K, Nolan T, Norman C, Provost L. The Improvement Guide: A Practical Approach to Enhancing Organizational Performance. San Francisco, CA: Jossey-Bass; 2009.

10. Schon D. The Reflective Practitioner: How Professionals Think in Action. New York: Basic Books; 1983.

11. Kitchenham A. The Evolution of John Mezirow's Transformative Learning Theory. Journal of Transformative Education. 2008;6(2):104-123. doi: $10.1177 / 1541344608322678$

12. Regan L, Hopson LR, Gisondi MA, Branzetti J. Learning to learn: A qualitative study to uncover strategies used by Master Adaptive Learners in the planning of learning. Med Teach. 2019;41(11):1252-1262. doi:10.1080/01 42159X.2019.1630729

13. Hunt D. Teacher's adaptation: "Reading and flexing to students. J Teach Educ. 1976;27(3):268-275. doi:10.1177/002248717602700323 
14. Davis DA, Mazmanian PE, Fordis M, Van Harrison $\mathrm{R}$, Thorpe KE, Perrier L. Accuracy of physician self-assessment compared with observed measures of competence: a systematic review. JAMA. 2006;296(9):1094-1102. doi:10.1001/ jama.296.9.1094

15. Friedman CP, Gatti GG, Franz TM, et al. Do physicians know when their diagnoses are correct? Implications for decision support and error reduction. J Gen Intern Med. 2005;20(4):334-339. doi:10.1111/j.15251497.2005.30145.x

16. Sargeant J, Armson H, Chesluk B, et al. The processes and dimensions of informed self-assessment: a conceptual model. Acad Med. 2010;85(7):1212-1220. doi:10.1097/ ACM.0b013e3181d85a4e

17. Price D, Swanson D, Irons M. Hawkins R. Longitudinal assessments in continuing specialty certification and lifelong learning. Medical Teacher. 2018;40(9): 917-919. doi:10.1080/01 42159X.2018.1471202

18. Krumholz HM. Big data and new knowledge in medicine: the thinking, training, and tools needed for a learning health system. Health Aff (Millwood). 2014;33(7):1163-1170. doi:10.1377/ hlthaff.2014.0053

19. Price DW, Wagner DP, Krane NK, et al. What are the implications of implementation science for medical education? Med Educ Online. 2015;20(1):27003. doi:10.3402/meo.v20.27003

20. Mylopoulos M, Brydges R, Woods NN, Manzone J, Schwartz DL. Preparation for future learning: a missing competency in health professions education? Med Educ. 2016;50(1):115123. doi:10.1111/medu.12893

21. Ross D, Morros M, Violato E. Critical Thinking skills of family physicians. Fam Med. 2020;52(9):635-641. doi:10.22454/ FamMed.2020.271478
22. Imprinting in Graduate Medical Education. ABFM Starfield Summit. https://static1.squarespace.com/static/5ef55bb3b8ab0958a88de0ac/t/ 5f4d5d2abb7ac34405573f52/1598905642414/ IssueBrief9-Imprinting.pdf. Accessed April 16, 2021

23. Nolen L. How medical education is missing the bull's eye. N Engl J Med 2020; 382:2489-2491. doi: 10.1056/NEJMp1915891.

24. Jetty A, Jabbarpour Y, Petterson S, Eden A Bazemore A. The declining presence of family physicians in hospital-based care. J Am Board Fam Med. 2019 Nov-Dec;32(6):771-772. doi: 10.3122/jabfm.2019.06.190152.

25. Starfield B, Shi L, Macinko J. Contribution of primary care to health systems and health. Milbank Q. 2005;83(3):457-502. doi:10.1111/ j.1468-0009.2005.00409.x

26. Hackbarth GM. Pursuit of an expanded physician supply. N Engl J Med. 2008;359(7):764766. doi:10.1056/NEJMc081123

27. Burrows H. Problem-based learning in medicine and beyond: a brief overview. New Dir Teach Learn. 1996;1996(68):3-12. doi:10.1002/ tl.37219966804

28. Hanna-Attisha M, LaChance J, Sadler R, Champney Schnepp A. Elevated Blood Lead Levels in Children Associated With the Flint Drinking Water Crisis: A Spatial Analysis of Risk and Public Health Response. AJPH Research. 2016. 106(2):283-290. doi: 10.2105/ AJPH.2015.303003

29. Yeager KA, Bauer-Wu S. Cultural humility: essential foundation for clinical researchers. Appl Nurs Res. 2013;26(4):251-256. doi:10.1016/j. apnr.2013.06.008

30. Pluck G, Johnson H. Stimulating curiosity to enhance learning, GESJ. Educ. Sci Psychol. 2011;2(19):24-31.
31. Crick R. Learning how to learn: the dynamic assessment of learning power. Curric J. 2007;18(2):135-153. doi:10.1080/09585170701445947

32. Hidi S, Renninger AK. The four-phase model of interest development. Educ Psychol. 2006;41(2):111-127. doi:10.1207/ s15326985ep4102_4

33. Neville A, Norman G. PBL in the undergraduate MD program at McMaster University: three iterations in three decades. Acad Med. 2007;82(4):370-374

34. Bransford JD, Schwartz DL. Rethinking transfer: A simple proposal with multiple implications. Rev Res Educ. 1999;24:61100.

35. Dweck C. Mindset: The New Psychology of Success. New York, NY: Ballantine Books; 2007.

36. Cutrer W, Pusic M, Gruppen L, Hammoud MM, Santen SA. The Master Adaptive Learner. Philadelphia, PA:, Elsevier; 2020.

37. Cutrer WB, Atkinson HG, Friedman E, Deiorio N, Gruppen LD, Dekhtyar M, Pusic M. Exploring the characteristics and context that allow master adaptive learners to thrive. Medical Teacher. 2018. 40:8. 791-796. doi: 10.1080/014 2159X.2018.1484560 